\title{
A Kalman Filter Technique to Estimate Relativistic Electron Lifetimes in the Outer Radiation Belt
}

D. Kondrashov, ${ }^{1,2}$ Y. Shprits, ${ }^{1}$ M. Ghil ${ }^{1,2,3}$ and R. Thorne, ${ }^{1}$

D. Kondrashov, Department of Atmospheric and Oceanic Sciences, 405 Hilgard Ave, Box 951565, 7127 Math Sciences Bldg. University of California, Los Angeles, CA, 90095-1565, U.S.A. (dkondras@atmos.ucla.edu)

${ }^{1}$ Department of Atmospheric and Oceanic

Sciences, University of California, Los

Angeles, CA 90095-1565, U.S.A.

${ }^{2}$ Institute of Geophysics and Planetary

Physics, University of California, Los

Angeles, CA 90095-1567, U.S.A.

${ }^{3}$ Département Terre-Atmosphère-Océan and Laboratoire de Météorologie

Dynamique (CNRS and IPSL), Ecole

Normale Supérieure, F-75231 Paris Cedex

05, FRANCE 
3 Abstract.

4

5

6

7

8

Data assimilation aims to smoothly blend incomplete and inaccurate observational data with dynamical information from a physical model, and become an increasingly important tool in understanding and predicting meteorological, oceanographic and climate processes. As space-borne observations become more plentiful and space-physics models more sophisticated, dynamical processes in the radiation belts can be analyzed using advanced data assimilation methods. We use the Extended Kalman filter and observations from the Combined Release and Radiation Effects Satellite (CRRES) to estimate the lifetime of relativistic electrons during magnetic storms in the Earth's outer radiation belt. The model is a linear parabolic partial differential equation governing the phase-space density. This equation contains empirical coefficients that are not well-known and that we wish to estimate, along with the density itself. The assimilation method is first verified on modelsimulated data, which allows us to reliably estimate the characteristic lifetime of the electrons. We then apply the methodology to CRRES measurements and show it to be useful in highlighting systematic differences between the parameter estimates for storms driven by coronal mass ejections (CMEs) and by corotating interaction regions (CIRs), respectively. These differences are attributed to the complex, competing effects of acceleration and loss processes during distinct physical regimes. The technique described herein may be applied next to constrain more sophisticated radiation belt and ring-current models, as well as in other areas of magnetospheric physics. 


\section{Introduction}

The radiation belts were discovered by Van Allen et al., [1958], but their structure is still poorly described, since satellite observations are often restricted to single-point measurements and thus have only limited spatial coverage. Therefore, to fill the spatio-temporal gaps in their description and thus lead to a better understanding of the dominant dynamical processes in the radiation belts, physics-based models should be combined with data in an optimal way. With more observational data coming from new and existing spacecraft, application of advanced data assimilation techniques finally becomes possible, by relying on the extensive experience with data assimilation in other geosciences [Bengtsson, 1975].

In the classical terminology of data assimilation [Bengtsson et al., 1981], the physical variables that characterize the state of the system under observation, and typically are functions of time and space, are referred to as state variables, especially in the case of a discrete state vector with only a few components, or as fields, when the space dependence is important and the state vector has a very large number $N$ of components; in numerical weather prediction, for instance, $N=\mathrm{O}\left(10^{6}-10^{7}\right)$. Determining the distribution of the state variables is usually referred to as state or field estimation. The evolution in time of the state or field variables is governed by a dynamical model, usually formulated as a discretized set of ordinary or partial differential equations. In a typical data assimilation scheme, the observational data and dynamically evolving fields are combined into the estimated fields by giving them weights that are inversely related to their relative errors or uncertainties. The fundamental properties of the system appear in the field equations as 
46

parameters. These parameters can be also included in the assimilation process; applying this approach to the radiation belts is the focus of the present study.

In this work, we will use the Kalman filtering algorithm [Kalman, 1960; Kalman and Bucy, 1961] to estimate the state of the radiation belts, given by the phase-space density (PSD) of relativistic electrons, and several parameters of a dynamic model that governs the evolution of the belts in time. The Kalman filter allows one to follow not only the evolution of the system's state and parameters, but it also propagates forward in time error estimates of state variables, thus naturally accounting for the system's evolving spatio-temporal uncertainties. For example, within a spatial region or during a time span in which the system is dynamically active, it is natural to expect the uncertainties of the estimated state to change fairly rapidly, compared to a "quiet regime," when and where these uncertainties might stay fairly constant. In the Kalman filter formulation, this information is readily provided by the dynamical evolution of time-dependent error covariance matrices. The use of a dynamical model is of fundamental importance in the Kalman filter, and sets it aside from other assimilation schemes and ad-hoc data analysis techniques.

The Kalman filter and its various generalizations have been successfully applied in various engineering fields and the geosciences, including autonomous or assisted navigation systems, as well as atmospheric, oceanic and coupled ocean-atmosphere studies [Ghil et al., 1981; Ghil and Malanotte-Rizzoli, 1991; Ghil, 1997; Sun et al., 2002], reanalysis of atmospheric data [Todling et al., 1998], and ionospheric modeling [Richmond and Kamide, 1988; Schunk et al., 2004]. This class of algorithms goes under the name of sequential filtering or sequential estimation and they are more and more widely used in operational 
69 
important loss processes, parameterized by the effective electron lifetimes, however, were not considered in their work and they used only synthetic observations. In contrast, our study uses real data from spacecraft observations in a more realistic radial diffusion model, which also accounts for the combined effect of local sources and losses. Moreover, we apply an extended Kalman filter to estimate model parameters that describe the net effect of source and loss processes, along with an estimation of the model state comprised of the relativistic-electron PSD.

The observational data are taken from the Combined Release and Radiation Effects Satellite (CRRES) spacecraft, for 100 consecutive days, starting on July 30, 1990. This time interval involves geomagnetic storms with distinctly different behavior: August 25, September 11 and October 9 in particular. Previous studies of these storms have provided evidence of the complex nature of competing loss and source processes that influence the radiation belts [Meredith et al., 2002; Brautigam and Albert, 2000; Iles et al., 2006]. The three main processes are pitch angle scattering into the atmosphere, radial diffusion, and energy diffusion, driven by various wave-particle interactions. In the absence of realistic time-dependent 3-D physical models to simulate these processes, various simpler approximations, such as radial transport models, are currently used instead.

Of particular interest is the estimation of the parameters of the acceleration and loss processes in such models. These parameters can be computed directly from a quasilinear theory by wave-particle interactions [Lyons et al., 1972; Abel and Thorne, 1998a,b]. They can be also estimated by analyzing the population of trapped and lost electrons in observational data [Thorne et al., 2005b; Selesnick et al., 2003, 2004; Selesnick 2006], or by relying on multiple model simulations with various parameter values, to obtain a 
better qualitative match with the observations [Brautigam and Albert, 2000; Shprits et al., 2005].

Selesnick et al. $[2003,2004]$ used least-square regression to estimate decay lifetimes that minimize the misfit between the observations and model-simulated data on electron pitchangle distributions. In contrast, we employ a radial diffusion model, while approximating the diffusion in pitch-angle and energy by an effective lifetime parameter, which accounts for the net effect of the loss and source processes. Also, we rely on the Kalman-filter approach that naturally combines the dynamically evolving uncertainties in both observations and the model, in order to obtain an estimate of electron lifetimes; this estimate is optimal within the sequential-estimation framework that we describe in Section 3 below. The results from both approaches will be compared in Section 5 .

In the next section, we summarize key properties of the radiation belts and describe the model used here to study their variability; the parameters that need to be estimated are introduced, too. In Section 3, we review the classical, linear Kalman filter for state estimation and the extended Kalman filter required by the nonlinear estimation of our model parameters. The results appear in Section 4, first for "identical-twin" experiments in which the true evolution of the system is known, and then for actual space-borne observational data. The conclusions and future work are discussed in Section 5.

\section{Data and Model}

\subsection{Outer Radiation Belt Variability}

${ }_{133}$ The radiation belts consist of electrons and protons trapped by Earth's magnetic field [Schulz and Lanzerotti, 1974]. Energetic protons form a single radiation belt, being confined to altitudes below $4 R_{E}$, where $R_{E}=6400 \mathrm{~km}$ is the nominal Earth radius. Electrons, 
on the other hand, exhibit a two-belt structure. The inner electron belt is located typically between 1.2 and $2.0 R_{E}$, while the outer belt extends from 4 to $8 R_{E}$. The quiet-time region of lower electron fluxes, between 2 and $3 R_{E}$, is commonly referred to as the "slot" region. The inner belt is very stable and is formed by slow inward diffusion from the outer radiation zone, subject to losses due to Coulomb scattering and losses to the atmosphere due to to pitch angle scattering by whistler-mode waves [Lyons and Thorne, 1973; Abel and Thorne, 1998a,b]. Relativistic electron fluxes in the outer radiation belt are highly variable; this variability is due to the competing effects of source and loss processes, both of which are forced by solar-wind-driven magnetospheric dynamics.

The adiabatic motion of energetic charged particles in the Earth's radiation belts can be described by guiding center theory [Roederer, 1970], and consists of three basic periodic components: gyro-motion about the Earth's magnetic field lines, the bounce motion of the gyration center up and down a given magnetic field line, and the azimuthal drift of particles around the Earth, perpendicular to the meridional planes formed by the magnetic polar axis and the magnetic field lines. There are three adiabatic invariants, each associated with one of these motions: $\mu, J$, and $\Phi$, respectively. Since adiabatic invariants are canonical variables [Landau and Lifshits, 1976], we can describe the evolution of the particles PSD in terms of these invariants and the corresponding phases, instead of the more usual space and momentum coordinates. By averaging over the gyro, bounce and drift motions, the PSD description can be reduced to describing the evolution of the adiabatic invariants only.

Each adiabatic invariant can be violated when the system is subject to fluctuations on time scales comparable to or shorter than the associated periodic motion [Schulz and 
Lanzerotti, 1974]. In the collisionless magnetospheric plasma, wave-particle interactions provide the dominant mechanism for violation of the invariants, and thus give rise to changes in radiation belt structure. Ultra Low-Frequency (ULF) waves have periods comparable to tens of minutes; the associated violation of $\Phi$ leads to radial diffusion. When the PSD of radiation belt particles exhibits a positive gradient with increasing radial distance, radial diffusion leads to a net inward flux and associated particle acceleration, provided that the first two invariants, $\mu$ and $J$, are conserved. Since the power in ULF waves is considerably enhanced during magnetic storms [Mathie and Mann, 2000], radial diffusion is considered to be a potentially important mechanism to account for the acceleration of energetic electrons during storm conditions [Elkington et al., 2004; Shprits and Thorne, 2004; Shprits et al., 2006a]. However, during the storm's main phase, losses to the magnetopause and consequent outward radial diffusion may deplete the radiation belts and cause a very fast loss of electrons [Shprits et al., 2006b].

Extremely Low-Frequency (ELF) and Very Low-Frequency (VLF) waves cause a violation of the invariance of $\mu$ and $J$, leading to pitch-angle scattering to the atmosphere [Thorne and Kennel, 1971; Summers and Thorne, 2003], as well as local energy diffusion [Horne and Thorne, 1998; Summers et al., 1998; Miyoshi et al., 2003; Horne et al., 2003, 2005]. These processes provide effective losses and sources of relativistic electrons on time scales comparable to those of radial diffusion. During storm-time conditions, the power spectral density of ULF waves [Mann et al., 2004], as well as that of ELF and VLF waves [Meredith et al., 2000, 2003], are strongly enhanced, and all three adiabatic invariants are violated simultaneously. 
Figure 1a shows the daily averaged relativistic $(1 \mathrm{MeV})$ electron fluxes measured by the MEA magnetic electron spectrometer [Vampola et al., 1992] flown on the Combined Release and Radiation Effects Satellite (CRRES) mission, as a function of $L^{*}$-shell, for 100 days starting on July 30, 1990, i.e. on the day-of-year (DOY) 210. The variable $L^{*}$ is the distance (in Earth radii) in the equatorial plane, from the center of the Earth to the magnetic field line around which the electron moves at time $t$, assuming that the instantaneous magnetic field is adjusted adiabatically to a pure-dipole configuration. In this study, the simplified Tsyganenko [1989] T89 magnetic field model has been used to derive electron fluxes at a particular $L^{*}$ value (from now on, we drop the superscript and refer to this variable simply as $L$ ). The $K p$ and $D s t$ indices are commonly used as proxies for geomagnetic activity and are shown in Fig. 1b,c; the data are taken from the World Data Center for Geomagnetism in Kyoto, Japan, http://swdcdb.kugi.kyotou.ac.jp/aedir/. The T89 model is specified by Kp and is valid only for relatively modest activity levels. Recent improved models of magnetic field include parameterization by Dst and solar wind measurements, though the latter is not generally available for the CRRES time period.

The black curve in Fig. 1a is the estimated position of the plasmapause, i.e. of the outer boundary of the plasmasphere; the latter is a region of the inner magnetosphere that contains relatively cool (low-energy) and dense plasma, populated by the outflow of ionospheric plasma along the magnetic field lines. The plasmapause position $L_{p p}$ can be approximately estimated, according to Carpenter and Anderson [1992], by

$$
L_{p p}=5.6-0.46 K p(t)
$$


where $K p(t)$ is the maximum of $K p$ over the $24 \mathrm{hr}$ preceding $t$. As described in Section 3 below, distinct loss processes operate inside and outside of the plasmasphere, and so we account for them separately in the physical model.

Even though relativistic electron fluxes in the outer belt are highly variable, flux enhancements occur over a broad range of $L$-values $(3.5 \leq L \leq 6.5)$, suggesting that a global acceleration mechanism operates over most of this belt [Baker et al., 1994]. During the period under study there were two very strong storms, as seen in Fig. 1a for $235 \leq t \leq 240$ DOY (August 26 storm), and $282 \leq t \leq 290$ DOY (October 9 storm). These two storms are associated with coronal-mass ejections (CMEs); typically they last only for several days but still produce intensifications down to the slot region [Meredith et al. 2002; Brautigam and Albert, 2000]. There are also recurrent storms associated with high-speed solar wind streams that arise in corotating interaction regions (CIRs). These somewhat weaker storms may last for more than a week and produce flux increases with a 27-day periodicity; see, for instance, the episode at $255 \leq t \leq 280 \mathrm{DOY}$, including the September 11 storm [Meredith et al., 2002; Iles et al., 2006]), and at $t \approx 300$ DOY in Fig. 1a.

The response of the radiation belt fluxes to solar wind variability is still poorly understood. Reeves et al. [2003] showed that approximately half of all geomagnetic storms either result in a net depletion of the outer radiation belt or do not substantially change relativistic electron fluxes as compared to pre-storm conditions, while the remaining $50 \%$ result in a net flux enhancement. Losses result from the collisions of orbitally trapped electrons with neutral atmospheric particles. Electrons with mirror points for their bounce motion that lie below $100 \mathrm{~km}$ are lost from the magnetosphere on the time scale of a 
$\mathrm{X}-12$

KONDRASHOV ET AL: A KALMAN FILTER TECHNIQUE

Here the radial diffusion term describes the violation of the third adiabatic invariant of motion $\Phi$, and the net effect of sources and losses due to violations of the $\mu$ and $J$ invariants is modeled by a characteristic lifetime $\tau_{L}$.

The parameters $D_{L L}$ and $\tau_{L}$ of Eq. (2) depend on the background plasma density, as well as on the spectral intensity and spatial distribution of VLF and ULF waves; all of these conditions are extremely difficult to specify accurately from limited point measurements. In this study we adopt an empirical relationship for the radial diffusion coefficient $D_{L L}=$ $D_{L L}(K p, L)[$ Brautigam and Albert, 2000] throughout the outer radiation belt:

$$
D_{L L}^{M}(K p, L)=10^{(0.506 K p-9.325)} L^{10} .
$$

This empirical, data-derived parameterization quantitatively agrees in the interior of the radiation belts with the independent theoretical estimates of Perry et al. [2005]. 
The specification for $\tau_{L}$ is more complicated, due to several competing wave-particle interaction mechanisms. Inside the plasmasphere, losses are mostly due to scattering by hiss waves, magnetospherically reflecting whistlers and coulomb collisions [Lyons et al., 1972; Abel and Thorne, 1998a]; these loss effects lead to lifetimes on the scale of 5-10 days at $\mathrm{MeV}$ energies. Outside the plasmasphere, chorus emissions produce fast pitch angle scattering with lifetimes on the scale of a day [Horne et al., 2005; Albert, 2005; Thorne et al. , 2005b]. Electromagnetic ion cyclotron (EMIC) waves could provide even faster but very localized losses of electrons with energies $\geq 0.5 \mathrm{MeV}$ on the time scale of hours [Thorne and Kennel, 1971; Summers and Thorne, 2003; ; Jordanova et al., 2001].

In the present study we use two different lifetime parameterizations, inside and outside the plasmasphere; inside we assume a time-constant $\tau_{L I}$, while outside we take

$$
\tau_{L O}=\zeta / K p(t)
$$

The inner boundary for our simulation $f(L=1)=0$ is taken to represent loss to the neutral atmosphere below. The variable outer boundary condition on the PSD is obtained from the CRRES observations at $L=7$ [Shprits et al., 2006a].

Figures $2 \mathrm{a}-\mathrm{c}$ show simulated fluxes from the numerical solution of Eq. (2) using a few realistic values of the parameters $\zeta$ and $\tau_{L I}$ in Eq. (4) and $D_{L L}$ given by Eq. (3). It is quite obvious that not all features of the observations can be adequately captured by fixed model parameters, no matter what combination of parameter values we try. Model results with both $\zeta$ and $\tau_{L I}$ equal to 10 days (Fig. 2b) globally overestimate fluxes at all $L$, indicating that these values are unreasonably long. Simulations with $\zeta=3$ days and $\tau_{L I}=10$ or 20 days (Figs. 2a,c) predict better the locations of the peak fluxes and the inner boundary of the enhanced fluxes, but fail to reproduce the duration of many storms. 
These simulations show that better estimates of dynamical model parameters are very important for radiation belt modeling. Running the model many times to find a "best match" with observations, by using various parameter combinations, is not a practical way to achieve such estimates, since these combinations cannot be exhausted when the number of state variables or the number of parameters is large. The results in Fig. 2 thus indicate the need for more accurate, automated techniques of estimating the dynamical model parameters by using an optimized combination of data and models. The Kalman filter described in the next section is capable of providing such a combination.

\section{State and Parameter Estimation}

\subsection{State Estimation and the Kalman Filter}

The Kalman filter [Jazwinski, 1970; Gelb, 1974] combines measurements that are irregularly distributed in space and time with a physics-based model to estimate the evolution of the system's state in time; both the model and observations may include errors. The estimate of the system's trajectory in its phase space minimizes the mean-squared error. We describe here briefly the Kalman filter algorithm in discrete time, following Ghil et al. [1981] and Ide et al. [1997].

For a system of evolution equations, including discretized versions of a partial differential equation like Eq. (2), the numerical algorithm for advancing the state vector $\mathbf{x}$ from time $k \Delta t$ to time $(k+1) \Delta t$ is:

$$
\mathbf{x}_{k}^{f}=\mathbf{M}_{k-1} \mathbf{x}_{k-1}^{a} .
$$

Here $\mathbf{x}_{k}=x(k, \Delta t)$ represents a state column vector, composed of all model variables: for our radiation belt model (2) it is the PSD at numerical grid locations in $L$. The matrix 

are also perturbed by random noise $\epsilon_{k}^{o}$ :

$$
\mathbf{y}_{k}^{o}=\mathbf{H}_{k} \mathbf{x}_{k}^{t}+\epsilon_{k}^{o} .
$$
from observation locations to nearby numerical grid points.

The "system" or "model" noise $\epsilon$ accounts for the net errors due to inaccurate model physics, such as errors in forcing, boundary conditions, numerical discretization, and subgrid-scale processes. Commonly, the column vector $\epsilon$ is assumed to be a Gaussian white-noise sequence, with mean zero and model-error covariance matrix $\mathbf{Q}, E \epsilon_{k}=0$ and $E \epsilon_{k} \epsilon_{l}^{T}=\mathrm{Q}_{k} \delta_{k l}$, where $E$ is the expectation operator and $\delta_{k l}$ is the Kronecker delta.

The observations $\mathbf{y}_{k}^{o}$, where superscript "o" refers to "observed," of the "true" system

The observation matrix $\mathbf{H}_{k}$ accounts for the fact that usually the dimension of $\mathbf{y}_{k}^{o}$ is less than the dimension of $\mathbf{x}_{k}^{t}$, i.e. at any given time observations are not available for all numerical grid locations. In addition, $\mathbf{H}_{k}$ represents transformations that may be needed if other variables than the state vector are observed, as well as any required interpolation

The observational error $\epsilon^{o}$ includes both instrumental and sampling error. The latter is also called representativeness error and is often due to the measurements being taken 
$\mathbf{P}_{k}^{a}=\left(\mathbf{I}-\mathbf{K}_{k} \mathbf{H}_{k}\right) \mathbf{P}_{k}^{f}$.

$$
\mathbf{x}_{k}^{a}=\mathbf{x}_{k}^{f}+\mathbf{K}_{k}\left(\mathbf{y}_{k}^{o}-\mathbf{H}_{k} \mathbf{x}_{k}^{f}\right) .
$$

The assumptions about the model and observational noise allow us to follow the time evolution of the forecast-error and analysis-error covariance matrices,

$$
\mathbf{P}_{k}^{f, a} \equiv E\left(\mathbf{x}_{k}^{f, a}-\mathbf{x}_{k}^{t}\right)\left(\mathbf{x}_{k}^{f, a}-\mathbf{x}_{k}^{t}\right)^{T}
$$

this evolution is given by

$\mathbf{P}_{k}^{f}=\mathbf{M}_{k} \mathbf{P}_{k-1}^{a} \mathbf{M}_{k}^{T}+\mathbf{Q}_{\mathbf{k}}$, 
The optimal gain matrix $\mathbf{K}_{k}$ in Eq. (9) is computed by minimizing the analysis error variance $\operatorname{tr} \mathbf{P}_{k}^{a}$, i.e. the expected mean-square error between analysis and the true state. This Kalman gain matrix represents the optimal weights given to the observations in updating the model state vector:

$$
\mathbf{K}_{k}=\mathbf{P}_{k}^{f} \mathbf{H}_{k}^{T}\left(\mathbf{H}_{k} \mathbf{P}_{k}^{f} \mathbf{H}_{k}^{T}+\mathbf{R}_{k}\right)^{-1}
$$

Equations (11) show that, after an update step, the analysis errors $\mathbf{P}_{k}^{a}$ are reduced [Ghil et al., 1981; Ghil, 1997]. Moreover, Eq. (12) shows that the variances of the forecast and the observations are weighted, roughly speaking, in inverse proportion to their respective variances [Ghil and Malanotte-Rizzoli, 1991]. The Kalman filter minimizes the expected error over the entire time interval, even though, due to its sequential nature, the observations are discarded as soon they are assimilated. When no observations are available at time $k$, only the forecast step is performed and

$$
\mathbf{P}_{k}^{a}=\mathbf{P}_{k}^{f}
$$

The Kalman gain is optimal when both the observational and model noise are Gaussian. If this is not so, which is quite likely in our case, then the Kalman gain will be suboptimal. Still, the identical-twin experiments in Section 4.1 demonstrate that, even in this case, we can obtain reliable and robust estimates of both the state and parameters.

\subsection{Parameter Estimation and the Extended Kalman Filter}

The Kalman gain $\mathbf{K}_{k}$ is optimal for a linear system, when both $\mathbf{M}(\mathbf{x})=\mathbf{M x}$ and $\mathbf{H}(\mathbf{x})=\mathbf{H x}$, as in Eqs. (5)-(7); in this case, under the assumptions mentioned in Section 3.1, the gain is based on the correct estimation of forecast error covariances from initial 


$$
(\tilde{\mathbf{M}})_{i j}=\frac{\partial M^{i}}{\partial x^{j}},(\tilde{\mathbf{H}})_{i j}=\frac{\partial H^{i}}{\partial x^{j}}
$$

here indices $i$ and $j$ refer to a particular matrix and state vector entry. The full nonlinear model is still used to advance the state. The EKF is first-order accurate in many situations but may diverge in the presence of strong nonlinearities [Miller et al., 1994; Chin et al., $2006]$.

A practical way to include estimation of model parameters into the Kalman filter is by the so-called state augmentation method [Gelb, 1974; Galmiche et al., 2003; Kao et al. 2006], in which the parameters are treated as additional state variables. For simplicity, let us assume that there is only one model parameter $\mu$ (not to be confused with the adiabatic invariant of motion): $\mathbf{M}=\mathbf{M}(\mu)$. By analogy with Eqs. (5) and (6), we can define equations for evolving the parameter's "forecast" and "true" values, by assuming, in the absence of additional information, a persistence model:

$\mu_{k}^{f}=\mu_{k-1}^{a}$

$\mu_{k}^{t}=\mu_{k-1}^{t}+\epsilon_{k}^{\mu}$

When additional information is available, Eq. (15) can be generalized to allow for more complex spatial and temporal dependence; such dependence may include, for instance, a seasonal cycle (e.g., Kondrashov et al. [2005]). 
Next, we form an augmented state vector $\overline{\mathbf{x}}$, model $\overline{\mathbf{M}}$ and error $\bar{\epsilon}$ :

$$
\overline{\mathbf{x}}=\left(\begin{array}{c}
\mathbf{x} \\
\mu
\end{array}\right), \overline{\mathbf{M}}=\left(\begin{array}{cc}
\mathbf{M}(\mu) & 0 \\
0 & 1
\end{array}\right), \bar{\epsilon}=\left(\begin{array}{c}
\epsilon \\
\epsilon^{\mu}
\end{array}\right)
$$

$$
\begin{aligned}
\overline{\mathbf{P}}_{k}^{f} & =\overline{\mathbf{M}}_{k}^{T} \overline{\mathbf{P}}_{k-1}^{a} \overline{\mathbf{M}}_{k}+\overline{\mathbf{Q}}_{k}, \\
\overline{\mathbf{K}}_{k} & =\overline{\mathbf{P}}_{k}^{f} \overline{\mathbf{H}}_{k}^{T}\left(\overline{\mathbf{H}}_{k} \overline{\mathbf{P}}_{k}^{f} \overline{\mathbf{H}}_{k}^{T}+R_{k}\right)^{-1}
\end{aligned}
$$

$$
\mathbf{y}_{k}^{o}=\left(\begin{array}{ll}
\mathbf{H} 0
\end{array}\right)\left(\begin{array}{c}
\mathbf{x}_{k}^{t} \\
\mu_{k}^{t}
\end{array}\right)+\epsilon_{k}^{0}=\overline{\mathbf{H}} \overline{\mathbf{x}}_{k}^{t}+\epsilon_{k}^{0}
$$

The Kalman filter equations for the augmented system become:

The analysis step for the augmented system involves only observations of the state:

$$
\overline{\mathbf{x}}_{k}^{a}=\overline{\mathbf{x}}_{k}^{f}+\overline{\mathbf{K}}_{k}\left(\mathbf{y}_{k}^{o}-\mathbf{H} \mathbf{x}_{k}^{f}\right)
$$

while the augmented error-covariance matrices involve cross-terms between the state variables and the parameter. Dropping from now on the time subscript $k$, we have

$$
\overline{\mathbf{P}}^{f, a}=\left(\begin{array}{ll}
\mathbf{P}_{x x}^{f, a} & \mathbf{P}_{x \mu}^{f, a} \\
\mathbf{P}_{\mu x}^{f, a} & \mathbf{P}_{\mu \mu}^{f, a}
\end{array}\right)
$$

Using the definition of $\overline{\mathbf{H}}$ in Eq. (18), we obtain:

$$
\overline{\mathbf{K}}=\left(\begin{array}{c}
\mathbf{P}_{x x}^{f} \mathbf{H}^{T} \\
\mathbf{P}_{\mu x}^{f} \mathbf{H}^{T}
\end{array}\right)\left(\mathbf{H} \mathbf{P}_{x x}^{f} \mathbf{H}^{T}+\mathbf{R}\right)^{-1}
$$

The augmented model propagates the forecast error of the parameter into the crosscovariance term $\mathbf{P}_{\mu x}^{f}$. By substituting Eq. (22) into Eq. (20), we can readily see that this 
error propagation enables the EKF to extract information about the parameter from the state observations and to update the unobserved parameter at the analysis step:

$$
\mu^{a}=\mu^{f}+\mathbf{P}_{\mu x}^{f} \mathbf{H}^{T}\left(\mathbf{H} \mathbf{P}_{x x}^{f} \mathbf{H}^{T}+\mathbf{R}\right)^{-1}\left(\mathbf{y}^{o}-\mathbf{H} \mathbf{x}^{f}\right) .
$$

This formulation can be easily extended to the case when several unknown parameters have to be estimated and $\mu$ then becomes a vector instead of a scalar [Ghil, 1997].

We apply the Kalman filter to estimate the lifetime parameters $\tau_{L I}$ and $\zeta$ in Eqs. (2) and (4). We did try to estimate $\tau_{L O}$ directly as well, but experiments with synthetic data (similar to those described in Section 4.1), showed that successful estimation of $\tau_{L O}$, along with $\tau_{L I}$, requires observations at a greater resolution in time than available in the CRRES data.

While the model in Eq. (2) is linear in PSD, the augmented system, including the lifetime parameters, is nonlinear because of the loss term, in which $\tau_{L}$ divides the PSD $f(L, t)$; therefore our sequential estimation problem becomes nonlinear. An additional nonlinearity arises due to the time-dependent position of the plasmapause boundary, as we will see in the next section. We adopt, therefore the EKF approach, and linearize $\overline{\mathbf{M}}$ (as in Eq. 14) around the current values of the augmented state vector formed by the PSD state vector and the two parameter values, $\tau_{L I}$ and $\zeta$.

It is well known (e.g. Richtmyer and Morton, 1967) that an implicit numerical scheme is best in order to solve a "stiff" parabolic partial differential equation, like Eq. (2), with diffusion coefficients that vary rapidly in space and time; see Eq. (3). For such problems, to achieve a given accuracy, it usually takes less computational time to use an implicit method with larger time steps than the explicit scheme, which requires much smaller time steps. For our implicit scheme, linearization with respect to the PSD is readily available 
KONDRASHOV ET AL: A KALMAN FILTER TECHNIQUE

and it follows from the known coefficients of M. Linearization with respect to the two lifetime parameters is more complex, because $\overline{\mathbf{M}}$ depends implicitly on the location of the plasmapause. We thus use small perturbations in the parameter values on the right-hand side of Eq. (2) and then apply numerical differentiation.

\section{Results and Discussion}

\subsection{Identical-Twin Experiments}

To test the parameter estimation scheme described in Section 3.2, we first conduct identical-twin experiments in which both the "true" solution, from which observations are drawn, and the forecast are produced by the same model, but with different lifetime parameter values. We obtain our "true" electron fluxes from a model run with $\tau_{L I}=20$ and $\zeta=3$ days (see Fig. 2a), and form synthetic observations by taking daily averages.

Our goal is to recover the "true" parameter values by assimilating observations into a model with the "incorrect" parameters: $\tau_{L I}=10$ and $\zeta=10$ days (see Fig. 2b). Numerical sensitivity experiments (not shown) confirm that other combinations of "true" and "incorrect" parameter values did not produce any adverse effects on the convergence of the parameter estimation process.

We start the forecast model with incorrect parameter values and non-zero model error $\epsilon_{\mu}$. The weights used in updating the parameters are related to the model errors assigned to the parameters; see Eqs. (16)-(23). The model error in the parameters should be chosen according to how much variation we are willing to allow the estimated parameters to have, and also how much information is needed from the observations. Since a smooth estimation of the parameters is often required, small error values tend to be a good choice: 
here we used $2 \%$ of their initial values. Data was assimilated only at $L \leq 5$ to avoid large uncertainties associated with higher $L$-values.

In the standard formulation of the Kalman filter, the noise covariances $\mathbf{Q}$ and $\mathbf{R}$ are assumed to be known [Jazwinski, 1970; Gelb, 1974]. This rarely happens in practice and usually some simple approximations are made [Dee et al., 1985]. For this study, both $\mathbf{Q}$ and $\mathbf{R}$ are assumed to be diagonal. Local values of the observation and model errors are taken to be $10 \%$ of the variance of the observed time series and the model-simulated ones, respectively. This heuristic approach worked well in the present study. Further development of adaptive filters, which estimate $\mathbf{Q}$ and $\mathbf{R}$ from the data as well $[$ Dee, 1995], is an active area of research, and we expect to use them in future work on the radiation belts.

Figures 3a,c show both "true" and estimated lifetimes $\tau_{L I}$ and $\tau_{L O}$ for our identical-twin experiment; a 48-hr window is used in plotting $\tau_{L O}$ to avoid artificial spikes due to the high temporal variability of $K p$. The outer-belt lifetime $\tau_{L O}$ converges to its "true" value at $\approx 235$ DOY.

The convergence for $\zeta$, which ultimately determines $\tau_{L O}$ and is shown in Fig. 3b, seems to be influenced strongly by the time-dependent plasmapause position; see Eq. (1). The value of $\zeta$ quickly drops from 10 days to about 5 in the presence of a strong storm at the beginning of the simulation, when the plasmapause is located at $L \leq 4$ (see Fig. 1a). Subsequently, until $t \approx 230 \mathrm{DOY}$, the geomagnetic conditions are quieter, the plasmapause expands above $L=5$, and therefore $\zeta$ does not change much. Its estimated standard deviation - i.e., the square root of the $\mathbf{P}_{\zeta \zeta}^{\mathbf{f}}$ component of the analysis-error covariance matrix - gradually increases due to additive model error at each forecast 
day, and $\zeta=20$ days - to show that, even in this highly nonlinear problem, convergence does not signficantly depend on the initial values of the parameters. Figure 5a shows the estimated lifetimes $\tau_{L I}$ and $\tau_{L O}$, the latter being again averaged over a 48-hr window; the parameter $\zeta$ is shown in Fig. 5b, while the assimilated fluxes are displayed in Fig. 5c.

As in the case of the identical-twin experiment of Fig. 3, for the first 20 days it is $\tau_{L I}$ that changes by slowly increasing in value as the plasmasphere fills the region within which observations are being assimilated (Fig. 5a). The value of $\zeta$ changes little during this period, while its estimated error $\left[\mathbf{P}_{\zeta \zeta}^{a}\right]^{1 / 2}$ gradually increases due to the addition of model error at each forecast step. The situation changes with the arrival of a strong storm at $t \approx 235$ DOY, when both $\zeta$ and $\tau_{L I}$ adjust dramatically to reach their relatively constant values of $\zeta \approx 3$ and $\tau_{L I} \approx 8$ days.

Electron fluxes obtained through data assimilation are expected to be closer to their actual values than those resulting from either model simulations or observations alone, since the assimilation process uses both model and data, and it accounts for errors or uncertainties in both. This fact explains certain differences between the assimilated fluxes in Fig. 5c and those in either Fig. 1a or Fig. 2, even after the initial interval of parameter convergence, i.e. at $t \geq 235$ DOY.

For the remainder of the assimilation run $\tau_{L I}$ remains in a tight range of $7 \leq \tau_{L I} \leq 9$ days. The values of $\zeta$, on the other hand, undergo intriguing transitions. They increase slowly to $\zeta \approx 7$ days, when a moderate intensity storm starts around $t \approx 260$ DOY, and remain at that level until a strong storm at $t \approx 285$ DOY leads to downward adjustment to $\zeta \approx 3$ days. The variations of $\zeta$ within the interval $260 \leq t \leq 280$ DOY are even more 
apparent for $\tau_{L O}$, which becomes comparable in value to $\tau_{L I}$ at $t \approx 270$ DOY (see Fig. $5 a)$.

The two regimes of behavior in the outer belt, for $240 \leq t \leq 260$ DOY and $260 \leq t \leq 280$ DOY, may be associated with differences in lifetime parameters during CME- and CIRdriven storms. Another possible explanation for the increased values of both $\zeta$ and $\tau_{L O}$ during a CIR storm is the neglect of a local acceleration source in Eq. (2). Such a source may be active during CIR-driven storms, which are associated with increased convection of hot electrons with an energy of about $100 \mathrm{KeV}$ [Lyons et al., 2005]. If such a source is present and has not been included in the model, it could be effectively captured in data assimilation by smaller loss estimates.

Still, the local acceleration by whistler chorus waves is more effective at higher energies and higher pitch angles, and loss is more effective at lower energies and pitch angles, while we present results only for near-equatorial particles of fixed energy. Ultimately, to distinguish between losses and sources one can use theoretical estimates of the pitch angle and energy scattering rates [Horne at al., 2005; Shprits et al., 2006c] to parameterize the local source term and the lifetime parameter and include both in the estimation process. Using results for a modified version of Eq. (2) that would include such a source term, with various $L$-values and statistical models for plasma density [Sheeley et al., 2001] and wave intensity [Meredith et al., 2003], one may also attempt to estimate the radial dependence of the source, as well as the loss processes.

In general, lifetime estimates based on the EKF do depend on the assumed radial diffusion coefficients; see Eq. (3). These estimates will be most sensitive to the values of the radial diffusion coefficients where timescales for losses and radial transport are com- 
parable, around $L=4.5$. However, at higher $L$-values fast radial transport tends to make distribution flat (diffusion-dominated region), while at low $L$-shells losses take over radial diffusion (loss-dominated region). In the heart of the radiation belts, diffusion coefficients derived by Brautigam and Albert [2000] agree well with the theoretical estimates of Perry et al. [2005]. Diffusion coefficients can be included in the parameter estimation procedure, and we plan to investigate this possibility in the future.

\section{Conclusions}

Our approach to estimating relativistic electron lifetimes is based on recognizing that parameters of the phase-space density (PSD) model (2), just like the model state variables, are subject to uncertainties. In addition, using model parameters $\tau_{L I}$ and $\tau_{L O}$ that are constant may not be optimal when the system exhibits distinct physical regimes, like CIRand CME-driven storms in the radiation belts.

Our identical-twin experiments with the extended Kalman filter (EKF), using synthetic data (Figs. 3 and 4), show that model parameter estimation can be successfully included in the data assimilation process by using the "state augmentation" approach; the "incorrect" model parameters can be driven toward their "correct" values very efficiently by assimilating model state variables. Doing so reduces the error in electron fluxes, with respect to the usual approach, in which the state only is estimated, while the model parameters are kept constant. The methodology described and tested here is applicable to more sophisticated radiation belt and ring current models, as well as in other areas of magnetospheric physics. This methodology holds even greater promise for the use of multiple-satellite measurements, where using independent observations at different $L$-shells should allow to make parameter estimation more often, thus providing a finer temporal resolution. 
When applying the EKF to actual CRRES data, we obtained lifetimes inside the plasmasphere on the scale of 5-10 days, which is consistent with previous theoretical estimates [Lyons et al., 1972; Abel and Thorne, 1998]. Our results are also consistent with the independent studies of observational data by Selesnick and associates [2003, 2004, 2006], which do not depend on modeling assumptions concerning radial transport and sources. In general, the intensity of plasmasphere hiss and associated losses do depend on activity levels $(K p)$, while our parameterization for $\tau_{L I}$ does not. For low-activity periods, however, the decay rates in the plasmasphere are exponential and can indeed be fitted with a constant lifetime parameter $\approx 5$ days, dependent only on energy [Meredith et al., 2006].

Since chorus waves outside the plasmasphere produce both local acceleration and local loss, the lifetime parameter $\tau_{L O}$ introduced here should be interpreted as a combined effect of local sources and losses, due to resonant wave-particle scattering by various types of waves (e.g., chorus, EMIC, and possibly hiss waves in the plumes). Our simulations indicate that observations are best reproduced with an effective lifetime parameter $\tau_{L O}$ of 2-3 days, which is comparable to the estimates of Thorne et al. [2005b]. Furthermore, our results are consistent with a claim that net effect of sources and losses is different during CME- and CIR-dominated storms. Quantifying these differences in greater detail by using parameter estimation is left for future research, where we plan to use multiple satellites during different parts of the solar cycle and concentrate on more accurate parameterizations of electron lifetimes at various energies. These parameterizations may be used in particle tracing codes that account quite accurately for the transport of the particles, but cannot resolve the violations of the first and second adiabatic invariants, $\mu$ and $J$. 
$\mathrm{X}-28$

KONDRASHOV ET AL: A KALMAN FILTER TECHNIQUE

Acknowledgments. We are grateful to Geoff Reeves and Reiner Friedel who provided

CRRES data. Three anonymous referees helped improve the presentation. This research was supported by grants NNG04GN44G and NNXO6AB846, which are part of NASA's "Living With a Star" program.

\section{References}

Abel, B., and R. M. Thorne (1998a), Electron scattering loss in Earth's inner magnetosphere, 1, Dominant physical processes, J. Geophys. Res., 103, 2385.

Abel, B., and R. M. Thorne (1998b), Electron scattering loss in Earth's inner magnetosphere, 2, Sensitivity to model parameters, J. Geophys. Res., 104, 4627.

Albert, J. M. (2005), Evaluation of quasi-linear diffusion coefficients for whistler mode waves in a plasma with arbitrary density ratio, J. Geophys. Res., 110, A03218, doi:10.1029/2004JA010844.

Baker, D. N. et al. (1994), Satellite anomalies linked to electrons in magnetosphere, Eos, Trans. $A G U, 75,401$.

Bengtsson, L. (1975), Four-Dimensional Data Assimilation of Meteorological Observations, GARP Publ. Ser. No 15. World Met. Org./Intl. Council Sci. Unions, Geneva.

Bengtsson, L., M. Ghil, and E. Källén (Eds.) (1981), Dynamic Meteorology: Data Assimilation Methods, Springer-Verlag, New York/Heidelberg/Berlin, 330 pp.

Bourdarie, S., et al. (1996), Magnetic storm modeling in the Earth's electron belt by the Salammbô code, J. Geophys. Res., 101, 27,171.

Brautigam, D. H., and J. M. Albert (2000), Radial diffusion analysis of outer radiation belt electrons during the October 9, 1990, magnetic storm, J. Geophys. Res., 105, 291. 
Ghil, M., S. Cohn, J. Tavantzis, K. Bube, and E. Isaacson (1981), Applications of estimation theory to numerical weather prediction, Dynamic Meteorology: Data Assimilation Methods, L. Bengtsson, M. Ghil and E. Källén (Eds.), Springer Verlag, pp. 139-224.

Ghil, M., and P. Malanotte-Rizzoli (1991), Data assimilation in meteorology and oceanography. Adv. Geophys., 33, 141-266.

Ghil, M. (1997), Advances in sequential estimation for atmospheric and oceanic flows. $J$. Meteor. Soc. Japan, 75, 289-304.

Horne, R. B., and R. M. Thorne (1998), Potential wave modes for electron scattering and stochastic acceleration to relativistic energies during magnetic storms, Geophys. Res. Lett., 25, 3011.

Horne, R. B., R. M. Thorne, Y. Shprits, et al. (2005), Wave acceleration of electrons in the Van Allen radiation belts, Nature, 44\%, 227-230.

Ide, K., P. Courtier, M. Ghil, and A. Lorenc (1997), Unified notation for data assimilation: Operational, sequential and variational. J. Meteor. Soc. Japan, 75, 181-189.

Iles, R. et al. (2006), Phase space density analysis of the outer radiation belt energetic electron dynamics J. Geophys. Res., 111, A03204, doi:10.1029/2005JA011206,

Jazwinski, A. H. (1970), Stochastic Processes and Filtering Theory, Academic Press, New York, 376 pp.

Jordanova, V. K., et al. (2001), Modeling ring current proton precipitation by EMIC waves during the May 14-16, 1997 storm, J. Geophys. Res., 106, 7.

Kalman, R. E. (1960), A new approach to linear filtering and prediction problems, Trans. ASME, Ser. D: J. Basic Eng., 82, 35-45. 
KONDRASHOV ET AL: A KALMAN FILTER TECHNIQUE

Kalman, R. E., and R. S. Bucy (1961), New results in linear filtering and prediction theory. Trans. ASME, Ser. D: J. Basic Eng., 83, 95-108.

Kalnay, E., et al. (1996), The NCEP/NCAR 40-year reanalysis project, Bull. Amer. Met. Soc., $77,437-471$.

Kalnay, E. (2003), Atmospheric Modeling, Data Assimilation and Predictability. Cambridge Univ. Press, Cambridge/London, UK, 341 pp.

Kao, J., D. Flicker, K. Ide and M. Ghil (2006), Estimating model parameters for an impact-produced shock-wave simulation: Optimal use of partial data with the extended Kalman filter, J. Comput. Phys.,214 (2), 725-737, doi: 10.1016/j.jcp.2005.10.022.

Kondrashov, D., S. Kravtsov, and M. Ghil (2005), A hierarchy of data-based ENSO models, J. Climate, 18(21), 4425-4444.

Landau, L. D and E. M. Lifshits (1976), Mechanics (3rd ed.). London: Pergamon. Vol. 1 of the Course of Theoretical Physics.

Lyons, L. R., R. M. Thorne, and C. F. Kennel (1972), Pitch angle diffusion of radiation belt electrons within the plasmasphere, J. Geophys. Res., r7, 3455.

Lyons, L. R., and R. M. Thorne (1972), Parasitic pitch angle diffusion of radiation belt particles by ion-cyclotron waves. JJ. Geophys. Res., 77, 5608.

Lyons, L. R., and R. M. Thorne (1973), Equilibrium structure of radiation belt electrons, J. Geophys. Res., 78, 2142.

Lyons, L. R., D.-Y. Lee, R. M. Thorne, R. B. Horne, and A. J. Smith (2005), Solar windmagnetosphere coupling leading to relativistic electron energization during high-speed streams, J. Geophys. Res., 110, A11202, doi:10.1029/2005JA011254. 
Mann, I. R., T. P. O'Brien, and D. K. Milling (2004), Correlations between ULF wave power, solar wind speed, and relativistic electron flux in the magnetosphere: solar cycle dependence, J. Atmos. Sol. Terr. Phys., 66, 187.

Mathie, R. A., and I. R. Mann (2000), A correlation between extended intervals of ULF wave power and storm-time geosynchronous relativistic electron flux enhancements, Geophys. Res. Lett., 27, 3261.

Meredith, N. P., et al. (2000), The temporal evolution of electron distributions and associated wave activity following substorm injections in the inner magnetosphere, $J$. Geophys. Res., 105, 12,907.

Meredith, N. P., et al., Outer zone relativistic electron acceleration associated with substorm enhanced whistler mode chorus, J. Geophys. Res,, 10\%, (A7), 1144, doi:10.1029/2001JA900146, 2002.

Meredith, N. P., et al. (2003), Statistical analysis of relativistic electron energies for cyclotron resonance with EMIC waves observed on CRRES, J. Geophys. Res., 108, (A6), 1250, doi:10.1029/2002JA009700.

Meredith, N. P., R. B. Horne, S. A. Glauert, et al. (2006) Energetic outer zone electron loss timescales during low geomagnetic activity, J. Geophys. Res., 111, A05212, doi:10.1029/2005JA011516.

Miller, R. N., M. Ghil and F. Gauthiez (1994), Advanced data assimilation in strongly nonlinear dynamical systems, J. Atmos. Sci., 51, 1037-1056.

Miyoshi, Y. et al. (2003), Rebuilding process of the outer radiation belt during the 3 November 1993 magnetic storm: NOAA and Exos -D observations, J. Geophys. Res., 108, (A1), 1004, doi:10.1029/2002JA007542. 
Naehr S. M., F. R. Toffoletto (2005), Radiation belt data assimilation with an extended Kalman filter, Space Weather, 3, S06001, doi:10.1029/2004SW000121.

Perry K. L., M. K. Hudson, S. R. Elkington (2005), Incorporating spectral characteristics of Pc5 waves into three-dimensional radiation belt modeling and the diffusion of relativistic electrons, J. Geophys. Res., 110, A03215, doi:10.1029/2004JA010760.

Reeves, G. D. et al. (2003), Acceleration and loss of relativistic electrons during geomagnetic storms, Geophys. Res. Lett., 30(10), 1529, doi:10.1029/2002GL016513.

Richmond, A. D., and Y. Kamide (1998), Mapping electrodynamic features of the high latitude ionosphere from localized observations: Technique, J. Geophys. Res., 93, 57415759.

Richtmyer, R. D., and K. W. Morton (1967), Difference Methods for Initial-Value Problems (2nd ed.). Interscience, New York, 405 pp.

Rigler, E. J., D. N. Baker, and R. S. Wiegel (2004), Adaptive linear prediction of radiation belt electrons using the Kalman filter, Space Weather, 2, S03003, doi:10.1029/2003SW000036.

Roederer, J. G. (1970), Dynamics of Geomagnetically Trapped Radiation, Springer-Verlag, New York.

Rossi, B. and Olbert S (1970), Introduction to the Physics of Space, McGraw-Hill Book Company.

Schulz, M., and L. Lanzerotti (1974), Particle Diffusion in the Radiation Belts, Springer, New York.

Schunk, R. W., et al. (2004), Global assimilation of ionospheric measurements (GAIM). Radio Sci. 39 RS1S02, doi:10.1029/2002RS002794. 
Selesnick, R. S., and J. B. Blake (2000), On the source location of radiation belt relativistic electrons, J. Geophys. Res., 105, 2607.

Selesnick, R. S. , J. B. Blake, and R. A. Mewaldt (2003), Atmospheric losses of radiation belt electrons, J. Geophys. Res., 108, 1468, doi:10.1029/2003JA010160.

Selesnick, R. S., M. D. Looper, and J. M. Albert (2004), Low-altitude distribution of radiation belt electrons, J. Geophys. Res., 109, A11209, doi:10.1029/2004JA010611.

Selesnick, R. S. (2006), Source and loss rates of radiation belt relativistic electrons during magnetic storms, J. Geophys. Res., 111, A04210, doi:10.1029/2005JA011473.

Sheeley, B. W., M. B. Moldwin, H. K. Rassoul, R. R. Anderson (2001), An empirical plasmasphere and trough density model: CRRES observations J. Geophys. Res., 106(A11), 25631.

Shprits, Y., and R. M. Thorne (2004), Time dependent radial diffusion modeling of relativistic electrons with realistic loss rates, Geophys. Res., Lett., 31, doi:10.1029/2004GL019591, 2004.

Shprits, Y. Y., R. M. Thorne, G. D. Reeves, and R. Friedel (2005), Radial diffusion modeling with empirical lifetimes: Comparison with CRRES observations, Annales Geophys., 23, 1467-1471.

Shprits Y. Y., R. M. Thorne, R. Friedel, G. D. Reeves, et al. (2006a), Outward radial diffusion driven by losses at magnetopause, J. Geophys. Res., 111, A11214, doi:10.1029/2006JA011657.

Shprits Y. Y., R. M. Thorne, R. B. Horne, S. A. Glauert, et al. (2006b), Acceleration mechanism responsible for the formation of the new radiation belt during the 2003 Halloween solar storm, Geophys. Res., Lett., 33, L05104, doi:10.1029/2005GL024256. 
KONDRASHOV ET AL: A KALMAN FILTER TECHNIQUE

643

Shprits Y. Y., R. M. Thorne, R. B. Horne, D. Summers (2006c), Bounce-averaged diffusion coefficients for field-aligned chorus waves, J. Geophys. Res., 111, A10225, doi:10.1029/2006JA011725.

Summers D, and R. M. Thorne (2003), Relativistic electron pitch-angle scattering by electromagnetic ion cyclotron waves during geomagnetic storms, J. Geophys. Res., 108, (A4), 1143, doi:10.1029/2002JA009489.

Summers D., R. M. Thorne, and F. Xiao (1998), Relativistic theory of wave-particle resonant diffusion with application to electron acceleration in the magnetosphere, $J$. Geophys. Res., 103, 20,487.

Sun, C., Z. Hao, M. Ghil, and J. D. Neelin (2002), Data assimilation for a coupled ocean-atmosphere model. Part I: Sequential state estimation. Mon. Wea. Rev., 130, 1073-1099.

Thorne, R. M., and C. F. Kennel (1971), Relativistic electron precipitation during magnetic storm main phase, J. Geophys. Res., 76, 4446.

Thorne, R. M., R. B. Horne, S. A. Glauert, N. P. Meredith, et al. (2005a), The influence of wave-particle interactions on relativistic electron dynamics during storms, Interactions: New Perspecives from Imaging Inner Magnetosphere, Geophys. Monogr. Ser., 159, eds. J. Burch, M. Schulz and H. Spense.

Thorne, R. M., T. P. O’Brien, Y. Y. Shprits, et al. (2005b) Timescale for MeV electron microburst loss during geomagnetic storms, J. Geophys. Res., 110, A09202, doi:10.1029/2004JA010882.

Todling, R, S. E. Cohn, and N. S. Sivakumaran (1998), Suboptimal schemes for retrospective data assimilation based on the fixed-lag Kalman smoother, Mon. Wea. Rev., 
666

667

668

669

670

671

672

$1262274-2286$.

Tsyganenko, N.A. (1989), A magnetospheric magnetic field model with a warped tail current sheet, Planet. Space Sci., 37, 5-20.

Vampola, A. L., J. V. Osborne, and B. M. Johnson (1992), CRRES magnetic electron spectrometer AFGL-701-5A (MEA), J. Spacecr. Rockets, 29, 592-594.

Van Allen, J. A., G. H. Ludwig, E. C. Ray, and C. E. McIlwain (1958), Observation of high intensity radiation by satellites 1958 Alpha and Gamma, Jet Propul., 28, 588. 


\section{a) CRRES Observations}
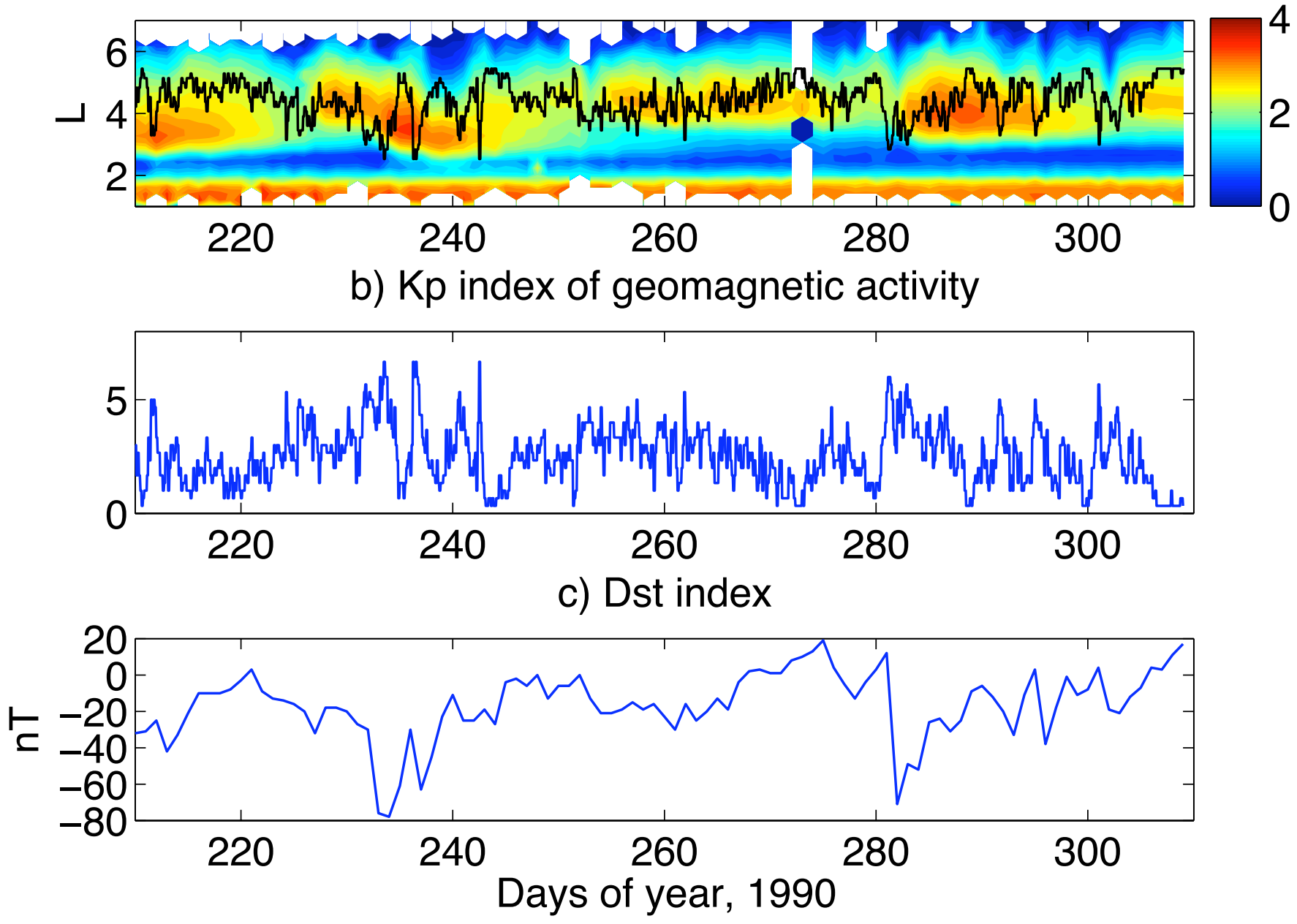

Figure 1. Radiation belt observations. (a) Daily averaged fluxes of electrons with an energy of $1 \mathrm{MeV}$, from CRRES satellite observations; values plotted are $\log _{10}$ (flux) in units of $\left(\mathrm{sr} \cdot \mathrm{keV} \cdot \mathrm{s} \cdot \mathrm{cm}^{2}\right)^{-1}$, with the black curve being the empirical plasmapause boundary [Carpenter and Anderson, 1992]. (b) Kp index (nondimensional), (this index is used to define the position $L_{p p}$ of the plasmapause in panel (a)), and (c) Dst index. Both indices are archived by the World Center for Geomagnetism (see text for details). 

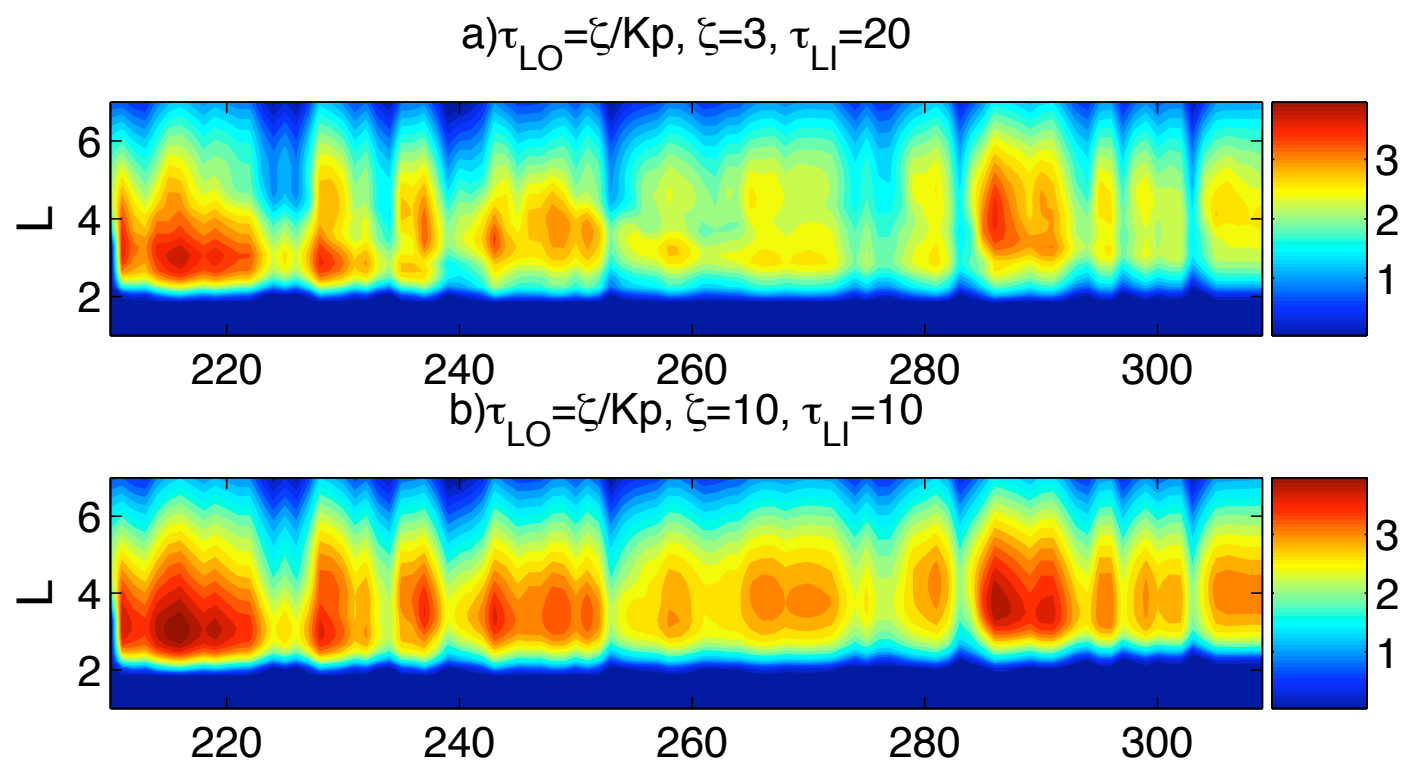

c) $\tau_{\mathrm{LO}}=\xi / \mathrm{Kp}, \zeta=3, \tau_{\mathrm{LI}}=10$

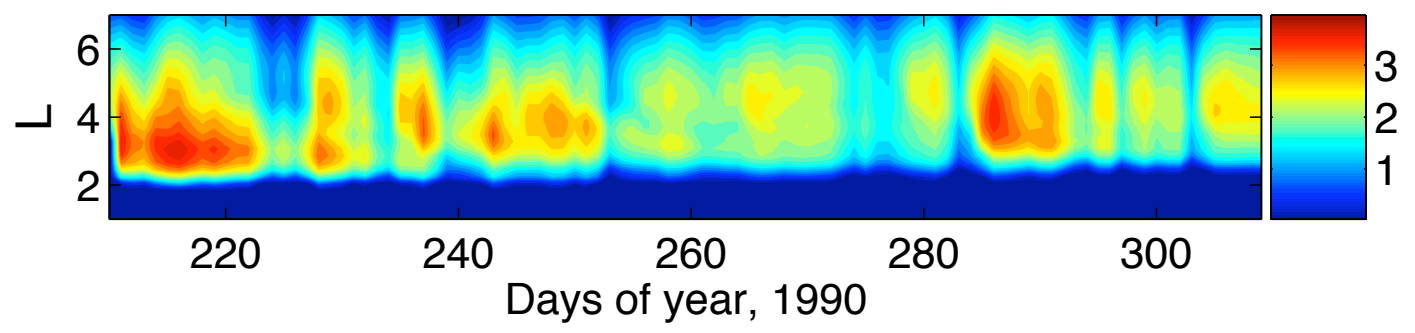

Figure 2. $\quad$ Simulated fluxes of $1-\mathrm{MeV}$ electrons, plotted as $\log _{10}(\mathrm{flux})$ in units of $\left(\mathrm{sr} \cdot \mathrm{keV} \cdot \mathrm{s} \cdot \mathrm{cm}^{2}\right)^{-1}$. The simulation uses different lifetime parameterizations outside $\left(\tau_{L O}=\right.$ $\zeta / K p(t))$ and inside $\left(\tau_{L I}\right)$ the plasmasphere: (a) $\tau_{L I}=20$ days, $\zeta=3$ days; (b) $\tau_{L I}=10$ days, $\zeta=10$ days; and (c) $\tau_{L I}=10$ days, $\zeta=3$ days. 

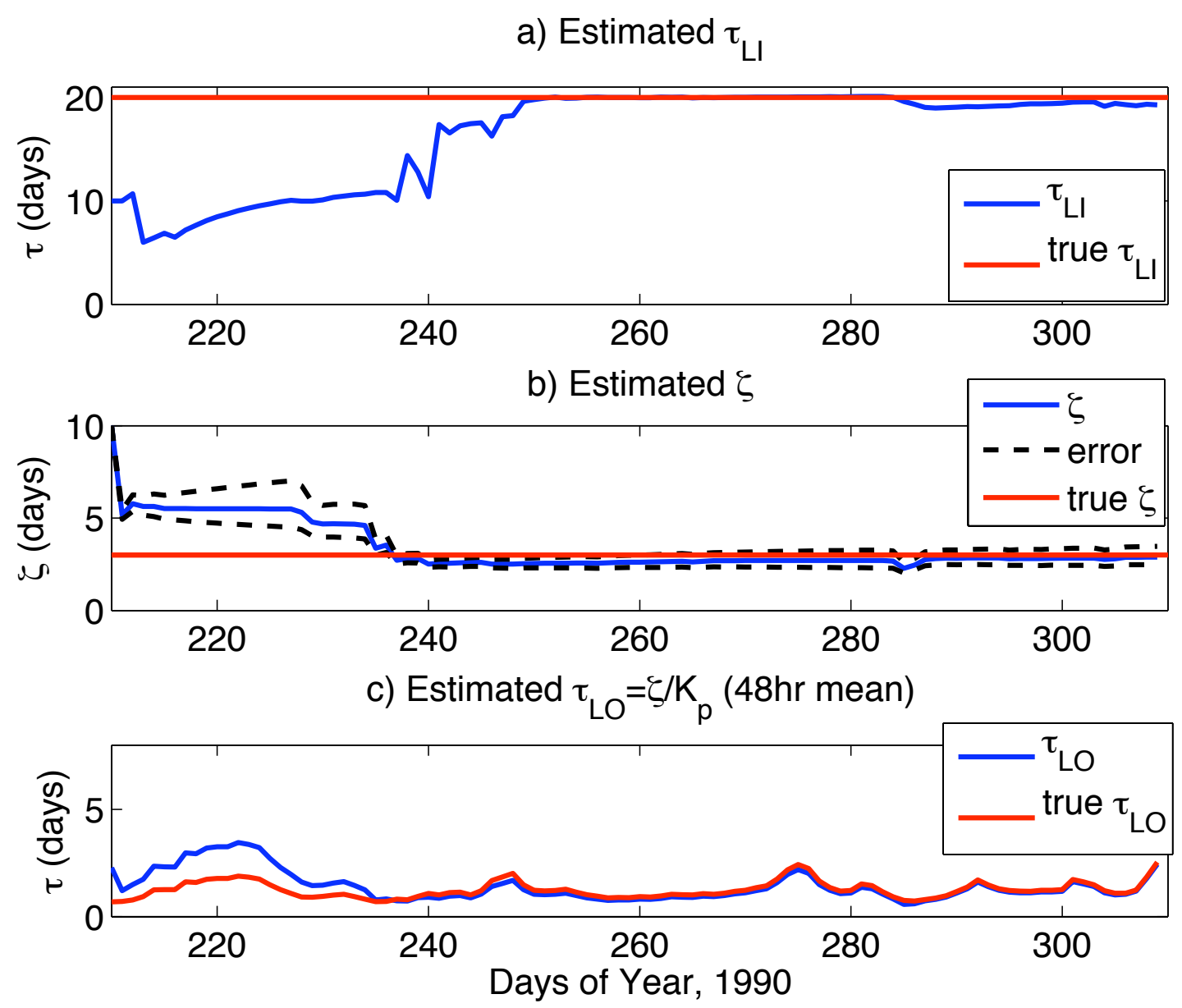

Figure 3. $\quad$ Parameter estimation in an identical twin-experiment: (a) $\tau_{L I}$; (b) $\zeta$ and its estimated uncertainty range $\left[\mathbf{P}_{\zeta \zeta}^{a}\right]^{1 / 2}$ (black dashed line); and (c) $\tau_{L O}=\zeta / K p$ (2-day running mean). Lifetimes are shown as estimated (blue line) and "true" (red line). 


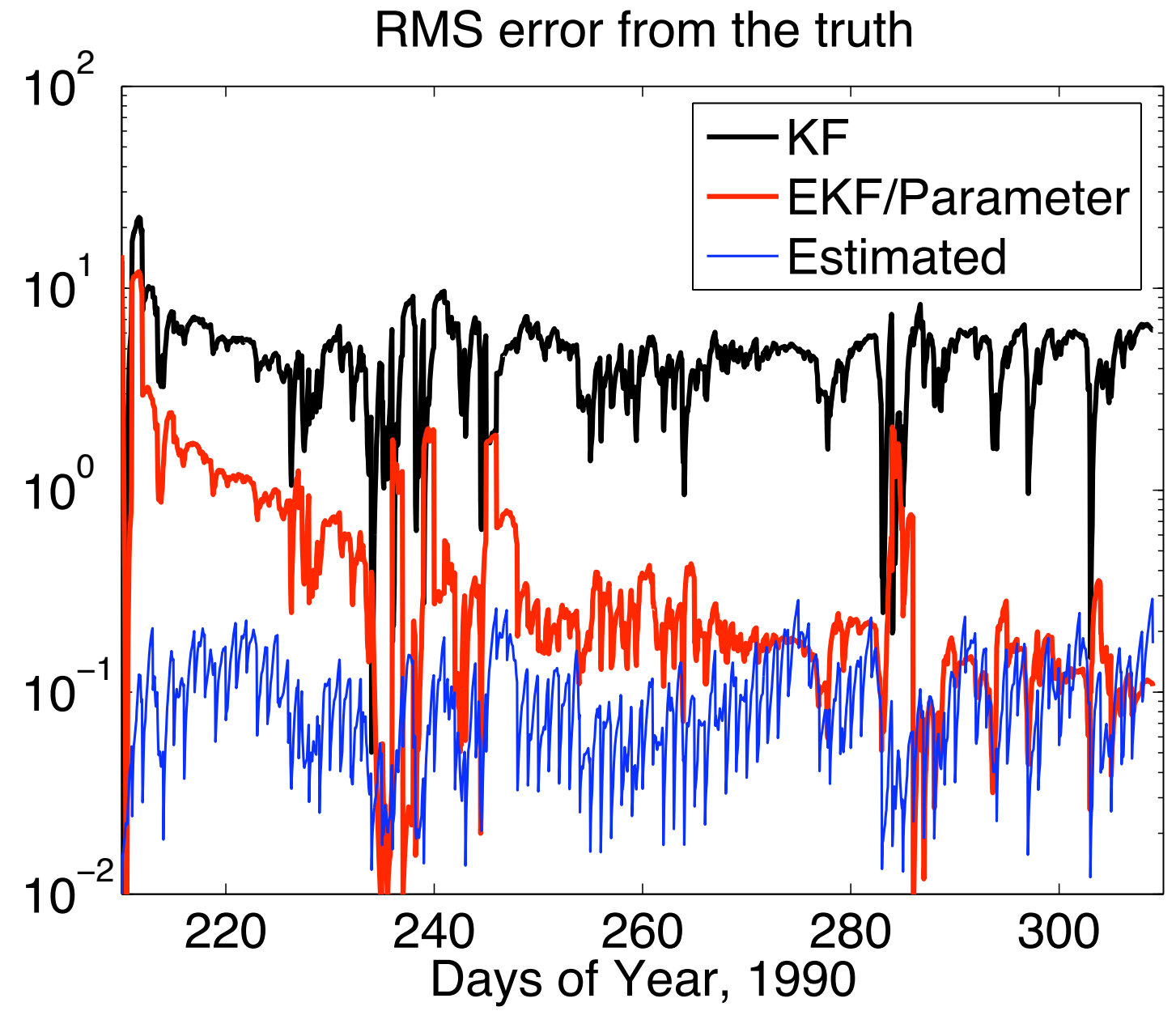

Figure 4. Root-mean-square (RMS) errors in the electron fluxes for the identical-twin experiment of Fig. 3. Black and red lines are for actual errors without and with parameter estimation, respectively; the blue line is an estimated error given by $\left[\operatorname{tr}\left(\mathbf{P}_{k}^{f}\right)\right]^{1 / 2}$. 
a) Estimated lifetimes

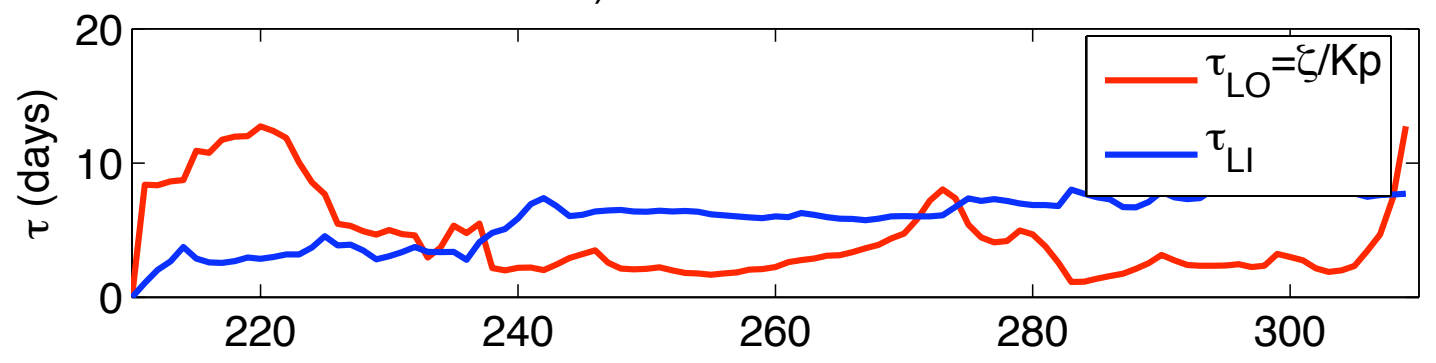

b) Estimated $\zeta$

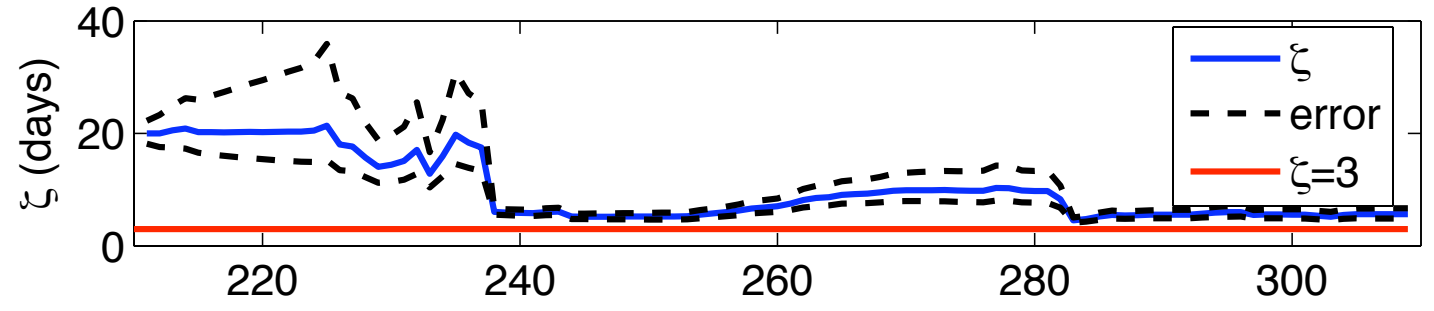

c) Estimated fluxes

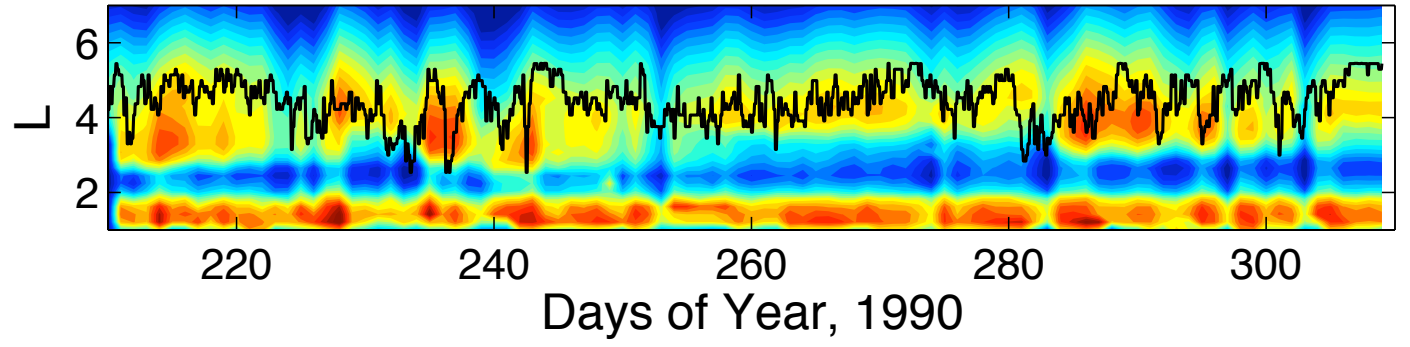

Figure 5. Results for parameter estimation with CRRES observations. (a) Estimated lifetimes: outside $-\tau_{L O}=\zeta / K p$ (2-day running mean, red line), and inside $-\tau_{L I}$ (black line) the plasmasphere; (b) $\zeta$ (blue line) and its estimated uncertainty range $\left[\mathbf{P}_{\zeta \zeta}^{a}\right]^{1 / 2}$ (black dashed line); and (c) daily $\log _{10}\left(\right.$ electron fluxes) at $1 \mathrm{MeV}$, in $\left(\mathrm{sr} \cdot \mathrm{keV} \cdot \mathrm{s} \cdot \mathrm{cm}^{2}\right)^{-1}$. In panel (c) the black solid line is the plasmapause and the color scale is the same as in Figs. 1a and 2. 